Questions vives

\section{Questions Vives}

Recherches en éducation

$N^{\circ} 25$ | 2016

L'activité des enseignants face au décrochage scolaire : Quelles difficultés et quelles mises en œuvre professionnelles?

\title{
Du décrochage provisoire au raccrochage scolaire : l'importance de la reconnaissance
}

From temporarily dropping out of school to getting back into the school system: the importance of recognition

\section{Liliane Pelletier et Driss Alaoui}

\section{(2) OpenEdition}

1 Journals

\section{Édition électronique}

URL : http://journals.openedition.org/questionsvives/1918

DOI : 10.4000/questionsvives. 1918

ISSN : 1775-433X

\section{Éditeur}

Université Aix-Marseille (AMU)

\section{Édition imprimée}

Date de publication : 7 juillet 2016

ISBN : 978-2-912643-49-0

ISSN : 1635-4079

\section{Référence électronique}

Liliane Pelletier et Driss Alaoui, « Du décrochage provisoire au raccrochage scolaire : l'importance de la reconnaissance », Questions Vives [En ligne], № 25 | 2016, mis en ligne le 02 juillet 2017, consulté le 30 avril 2019. URL : http://journals.openedition.org/questionsvives/1918; DOI : 10.4000/

questionsvives. 1918

Ce document a été généré automatiquement le 30 avril 2019.

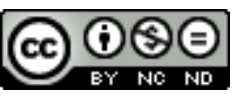

Questions Vives est mis à disposition selon les termes de la licence Creative Commons Attribution -

Pas d'Utilisation Commerciale - Pas de Modification 4.0 International. 


\section{Du décrochage provisoire au raccrochage scolaire : l'importance de la reconnaissance}

From temporarily dropping out of school to getting back into the school system: the importance of recognition

Liliane Pelletier et Driss Alaoui

\section{Introduction}

1 Le décrochage scolaire est une réalité complexe qui exige, pour être appréhendée, une double vigilance : scientifique et éthique. Les qualifications hâtives des élèves en tant que décrocheurs procèdent par réduction provoquant un enfermement d'individus dans des catégories dont les définitions et les indicateurs demeurent flous et peu précis. Plusieurs auteurs (Blaya, 2010 ; Costa-Lascoux, 2002 ; Glasman, 2000, 2014) ont souligné les limites et les effets d'une telle démarche, et proposent un renversement de l'ordre des priorités épistémiques. En effet, la focalisation sur les décrocheurs occulte le phénomène du décrochage scolaire et évacue le caractère multidimensionnel (institutionnel, politique, social...) pour ne retenir, finalement, que la dimension individuelle. Ce glissement d'un phénomène en mouvement, qui mérite davantage d'attention, vers une isolation par catégorisation qui fige et transforme le provisoire en trait définitif est susceptible de favoriser la stigmatisation. Plus grave, c'est qu'il risquerait, ainsi que le souligne Yves Lenoir (2012) au sujet du déni de reconnaissance, de conduire vers une prise en charge forcée d'un sujet qui ne demande rien. Nous avons jugé pertinent, dans le cadre de notre recherche, de laisser la place à une écoute plurielle des points de vue des élèves vivant des situations de décrochage scolaire.

2 Par ailleurs, l'étude du décrochage scolaire appelle celle du raccrochage scolaire, c'est cette unité articulant deux processus, qui sont totalement différents, mais 
complémentaires dans le sens où ils sont indispensables pour une meilleure compréhension de ce qui se défait et de ce qui se retisse, qui a été privilégiée dans la présente contribution. Si cette mise en lien nous semble pertinente sur le plan épistémologique, elle l'est également sur le plan éthique. L'étude du décrochage scolaire ne doit pas empêcher la prise en compte des efforts fournis par ceux qui se trouvent dans une situation de décrochage pour la dépasser. La logique dichotomique, même si elle pourrait être partiellement justifiée sur le plan méthodologique, nous a semblé en opposition avec notre conception de l'élève en tant que sujet complexe. C'est d'ailleurs pour ces raisons que l'entrée par le sujet a été retenue dans une perspective compréhensive. La dimension subjective, associée à d'autres dimensions, place en premier la question de sens et, par ricochet, le souci de parvenir à une intelligibilité du décrochage-raccrochage scolaire.

3 Nous présenterons dans une première partie, un rappel de l'ampleur du phénomène du décrochage scolaire et un descriptif d'un projet et dispositif de raccrochage scolaire. La seconde sera dédiée aux cadres théorique et conceptuel, qui seront mobilisés pour interpréter les données, ainsi qu'au cadre méthodologique. La troisième partie sera consacrée à l'analyse et à l'interprétation des résultats de la phase $\mathrm{n}^{\circ} 1$ (avant que les élèves n'intègrent le dispositif de raccrochage scolaire «Il faut tout un village pour éduquer un enfant »), et ceux de la phase $\mathrm{n}^{\circ} 2$ (après quatre mois de fréquentation $\mathrm{du}$ dispositif).

\section{Le décrochage scolaire : une préoccupation nationale et locale}

4 Si le décrochage n'est pas un phénomène nouveau, il constitue aujourd'hui une préoccupation à tous les niveaux de la société (responsables politiques, acteurs de la communauté éducative et scolaire, etc.). Dans les années 1970, on dénombrait 200000 élèves quittant le système scolaire sans aucune qualification. Situation certes préoccupante, mais ne provoquant pas pour autant, vu le contexte économique de l'époque, des réactions politiques et sociales vives. Aujourd'hui, les statistiques nous livrent des chiffres qui sont moins élevés que ceux des années 1970, environ 140000 décrocheurs, mais qui ont la particularité de faire l'objet de commentaires, d'analyses et de prises de décisions à tous les niveaux de l'Éducation nationale et d'autres ministères. Toutes ces réactions participent à la visibilisation du phénomène et attestent d'une grande mobilisation pour lutter contre le décrochage scolaire, un problème perçu comme social. Cependant, ainsi que l'explique à juste titre François Dubet (2014) :

l'émergence du problème du décrochage et sa transformation en problème social prioritaire résultent donc de l'impératif de réussite associé à l'égalité des chances et des effets de l'emprise croissante des diplômes sur l'entrée dans la vie professionnelle. Les élèves qui échouent, ceux qui décrochent et ceux qui échouent et décrochent, deviennent les symptômes d'une difficulté générale d'ajustement de la formation à l'emploi. (p. 11)

À l'île de La Réunion, où le taux de chômage de jeunes de moins de 25 ans est de 56 \%, la lutte contre le décrochage scolaire constitue une priorité majeure. En effet, le Conseil régional comme l'Académie et ses partenaires font de sorte qu'une situation provisoire ne devienne, pour certains élèves, définitive. C'est dans cette optique également que plusieurs établissements du second degré se sont lancés, en partenariat avec des 
chercheurs, dans des recherches-action et dans la mise en place de dispositifs de raccrochage scolaire. Nous nous limitons ici à présenter celui qui a été retenu pour la présente étude.

Dans le cadre de ce texte, nous ne présenterons qu'une partie des résultats de notre recherche, en nous limitant ici à une seule catégorie d'acteurs, les élèves et en nous centrant sur les deux questions de recherche suivantes:

7 Comment les élèves décrocheurs perçoivent-ils et vivent-ils les situations de décrochage scolaire?

En quoi l'engagement de ces élèves dans le dispositif « il faut tout un village pour éduquer un enfant" altère-t-il leurs perceptions initiales et enclenche-t-il un processus de raccrochage scolaire?

\section{Cadre théorique}

9 C'est donc en termes de processus, de mouvement, d'interaction, de donation de sens et de tensions qu'il convient d'appréhender les situations de décrochage et raccrochage scolaires. Dans cette optique, nous nous référons à une microsociologie interactionniste (De Queiroz \& Zolvotscki, 1994 ; Goffman, 1974 ; Lapassade, 1997, 1998 ; Le Breton, 2004 ; Thomas, 1984)

10 Nous nous appuyons également sur la théorie de la reconnaissance telle qu'elle est conçue par Axel Honneth (2010) en lien avec le processus de raccrochage scolaire.

\subsection{Microsociologie interactionniste}

Le décrochage et le raccrochage scolaires constituent un tout complexe renvoyant à différents niveaux de la réalité (micro, méso et macro) que Gurvitch (1963) a bien distingués sans toutefois les séparer. La typologie des niveaux proposée par Gurvitvh pourrait faciliter le nécessaire travail de contextualisation. Elle a le mérite de procéder par dialectisation permettant ainsi de percevoir la réalité dans sa dynamique, dans son engendrement et aussi dans son extinction. Cette conception a été reprise par des sociologues interactionnistes notamment par Strauss (1992).

Dans le cadre de la présente recherche, nous avons, sans oublier les autres niveaux de la réalité, choisi une entrée microsociologique. Dans cette perspective, nous avons convoqué l'interactionnisme symbolique pour appréhender et interpréter des données relatives au décrochage et au raccrochage scolaire. Ce courant repose sur trois principes présentés par De Queiroz et Ziolkovski M. (1994) :

1. les humains agissent à l'égard des choses en fonction du sens interprétatif que ces choses ont pour eux ;

2. ce sens est dérivé ou provient des interactions que chacun a avec autrui ;

3. c'est dans un processus d'interprétation mis en œuvre par chacun dans le traitement des objets rencontrés que ce sens est manipulé et modifié.

13 Comme on peut le remarquer, ces principes mettent l'accent sur la question de sens et sur la capacité des individus d'agir au sein de leur monde à partir du sens qu'ils confèrent à leur ici et maintenant. 

principes évoqués, est le concept de définition de la situation que nous avions jugé pertinent pour saisir et interpréter les points de vue des élèves engagés dans un processus de décrochage scolaire et que le dispositif mis en place par une équipe enseignante tente d'intégrer dans un processus de raccrochage scolaire.

Selon le théorème de Thomas, "quand les hommes définissent des situations comme réelles, elles sont réelles dans leurs conséquences ». Cela veut dire, ainsi que l'explique Javeau (2003), que les situations sociales sont fortement liées aux représentations et «n'ont pas d'autre réalité que celle qui leur est conférée, en raison d'une définition commune, par ceux et celles qui y prennent part» (p. 57). Dans cette perspective, le contexte, tel que le précise Lenoir (2013), n'est pas réductible à un contenant rempli par des sujets en interaction; il n'est pas non plus une réalité immuable (Ropé, 2002). Sans nier la dimension objective d'une situation, Thomas introduit le point de vue subjectif du sujet. Ainsi, le contexte est de fait construit par l'interaction et par l'interprétation d'une pluralité d'éléments par des sujets se référant à des cadres de référence qui ne sont pas immuables dès lors qu'ils subissent eux-mêmes, à chaque fois qu'ils sont convoqués, des micro-changements et des altérations.

En reconnaissant que chaque définition de la situation appelle des contre-définitions, Thomas n'exclut pas les tensions inter-définitions ce qui atteste aussi de la plasticité de la situation.

Par ailleurs, Goffman (1974) explique que les interactions s'inscrivent dans une pluralité de cadres ${ }^{1}$ qui s'emboîtent, mais qui se distinguent par leur degré de structuration. Ceuxci jouent un rôle important dès lors qu'ils permettent aux sujets de définir la situation et de se livrer à une activité interprétative dont l'une des conséquences est le caractère provisoire des définitions de la situation. Goffman soutient l'idée selon laquelle «toute définition de la situation est construite selon des principes d'organisation qui structurent les événements - du moins ceux qui ont un caractère social - et notre propre engagement subjectif. Le terme de « cadre » désigne ces éléments de base » (p. 19).

\subsection{Cadre conceptuel : éléments de définition}

\subsubsection{Le décrochage provisoire}

Dans son acception élémentaire, la notion de décrochage scolaire renvoie à une rupture scolaire sans l'obtention d'un diplôme. Si cette notion permet d'appréhender les 140000 élèves qui quittent le système scolaire sans qualification, elle reste toutefois muette concernant les élèves présents physiquement, mais absents cognitivement. Il s'agit d'une population difficilement quantifiable et dont le nombre serait beaucoup plus important que celui des décrocheurs. Il nous a semblé pertinent d'avancer dans la connaissance de cette catégorie qui relève de ce que nous nommons ici le « décrochage provisoire ». Dans un registre macrosociologique, Pierre Bourdieu et Patrick Champagne (1993) parlaient des « exclus de l'intérieur », ceux qui sont victimes d'une massification inachevée dès lors qu'elle n'a pas débouché sur une démocratisation permettant de réduire considérablement les effets des inégalités sociales dans le domaine scolaire.

De son côté, Glasman $(1998,2000)$ désigne ces élèves sous le vocable de « décrocheurs sur place » et soulève des questions pertinentes ${ }^{2}$ quant à la singularité de cette catégorie par rapport à ceux qui décrochent définitivement. Par ailleurs, Rochex (1998) nomme cette 
catégorie "décrocheurs passifs » tout en attirant l'attention sur le fait qu'elle pourrait masquer le caractère pluriel des processus conduisant vers le décrochage. Enfin, dans une recherche relative au décrochage à l'université, Sarfati (2013) avance la notion de « détachement scolaire ».

Par décrochage provisoire, nous entendons un entre-deux vulnérable parce qu'il est susceptible, selon les circonstances et les décisions prises, de basculer soit dans le décrochage définitif soit dans le raccrochage scolaire. Il exprime une démobilisation scolaire (Ballion, 1995), une baisse de motivation, un refus de participation, des absences répétées, des comportements relevant de la déviance scolaire, un manque de plaisir affectant progressivement les représentations de l'élève, son rapport à l'école, aux savoirs, à l'enseignant et à ses pairs et à soi-même. Ces changements sont des microruptures qui risquent de fragiliser les liens que l'élève tisse avec son entourage scolaire et familial. Ainsi, la détérioration de la qualité du lien pédagogique (Broccolichi, 2000) se dresse comme obstacle empêchant la réalisation de l'acte d'enseigner, d'apprendre et de socialiser. Dans cette perspective, le décrochage provisoire fonctionne comme un analyseur d'une situation complexe. Être présent tout en étant relativement absent intellectuellement signifie que l'école est devenue insupportable, elle l'est davantage quand l'humiliation, le mépris et le déni de reconnaissance (Alaoui, 2016) deviennent des réactions et/ou réponses aux effets du décrochage provisoire.

21 Le décrochage provisoire ne concerne pas un public particulier même si chez les élèves issus de milieux défavorisés le risque de décrocher est assez important. Il ne renvoie pas à des parcours identiques et s'il laisse voir quelques similitudes entre des élèves concernés, c'est en termes de singularité qu'il conviendrait d'appréhender les élèves engagés dans le processus de décrochage provisoire. La question de sens demeure donc centrale.

Il y a des élèves venant de milieu favorable et qui ne sont pas en situation d'échec et qui décrochent et des élèves en situation d'échec, mais qui ne décrochent pas (Glasman, 2004, 2014). C'est dans ce sens que les notions d'échec et de décrochage ne disent pas la même chose, comme le précise Glasman (2014), elles se recoupent, mais ne se superposent pas. Si nous avons opté pour la notion de décrochage provisoire c'est pour la simple raison que cette situation n'est pas définitive, elle n'est pas une fatalité et qu'elle peut être transformée en raccrochage scolaire. C'est cette notion qui sera traitée dans le paragraphe suivant.

\subsubsection{Le raccrochage scolaire}

Il constitue une réponse complexe à la situation de décrochage scolaire dans le sens où il ne se réduit pas à un simple traitement. Le traitement purement quantitatif qui n'implique pas l'élève en tant que sujet connaissant semble être un facteur d'aggravation qui pousse l'élève à prendre connaissance de l'impossibilité de surmonter les difficultés rencontrées. Si le raccrochage est d'ordre scolaire, il demeure multidimensionnel comme l'est d'ailleurs le décrochage. Et s'il s'adresse directement à l'élève, il concerne, à des degrés variables, l'ensemble des acteurs et tente de rendre centrale la question du sens et du rapport à l'école, aux savoirs, à l'enseignant, aux parents et à soi.

Cependant, deux préalables semblent être nécessaires pour enclencher le processus de raccrochage scolaire : la reconnaissance, qui fournit aux êtres humains les bases de la dignité et de l'estime et sans laquelle il devient difficile d'exister (Caillé, 2004) et la 
coconstruction d'une définition commune et partagée de la situation. Ces éléments conditionnent la mobilisation scolaire l'un des signes du raccrochage scolaire.

\subsubsection{Le raccrochage scolaire et la reconnaissance} celle du raccrochage scolaire. Ce dernier signifie et renvoie à une convergence d'efforts : efforts collectifs (écoute, concertation, dialogue, décloisonnement des frontières, absence d'accusations mutuelles, passage d'une démarche reposant sur la prise en charge vers un accompagnement...) consentis par différents acteurs de la communauté scolaire et éducative et efforts individuels associés à une volonté de surmonter la situation de décrochage scolaire (assiduité, respect des normes, implication...). Par ailleurs, le raccrochage est un processus qui repose, entre autres, sur la reconnaissance du sujet en tant que personne singulière. L'expérience négative du décrochage nous renseigne sur l'importance du raccrochage et de la réussite comme les dénis de reconnaissances sont révélateurs de l'importance de la reconnaissance. En se référant aux travaux de Hegel relatifs à la reconnaissance, Honneth (2010) précise que la reconnaissance consiste en une confirmation intersubjective par autrui des capacités et des valeurs qu'un sujet, individuel ou collectif, considère comme siennes et auxquelles il accorde une importance particulière. Selon Honneth, la reconnaissance est due

à la combinaison de deux éléments : l'identification cognitive et l'expression. Dans un premier temps, une certaine personne est connue en tant qu'individu avec des qualités particulières, selon les données de la situation, et, dans un second temps, cette connaissance reçoit une expression publique par le fait que l'existence de la personne perçue est confirmée aux yeux des personnes présentes par des actes, des gestes ou des expressions du visage (Honneth, 2006, p. 231).

En s'appuyant sur ces considérations, Honneth (2010) distingue trois sphères de reconnaissance, trois types de relation à soi et trois principes déterminant les attentes légitimes de chaque sujet humain. fusion originelle entre la mère et son enfant que la séparation vient briser pour permettre à l'enfant d'accéder au statut de personne indépendante. Cependant, cette indépendance, qui signifie la capacité d'être seul sans être inquiet, et son développement sont conditionnés par la certitude de préserver l'amour d'autrui et de rester un être aimé. C'est ainsi que l'individu parvient à avoir une confiance en soi et élaborer un rapport positif à lui-même. En effet, "Sans la conviction profonde que la personne aimée lui restera attachée même après avoir recouvré son indépendance, le sujet aimant ne serait pas en mesure de lui reconnaître son nouveau statut " (Honneth, 2010, p.131). Cette reconnaissance s'étend à d'autres contextes relationnels et à d'autres types de relation tels que l'amitié, le lien familial, etc.

\section{Le principe d'égalité et le respect de soi dans la sphère des relations juridiques}

Le principe de réciprocité se donne également à voir avec force dans la reconnaissance juridique. Celle-ci, selon Honneth (2010), signifie qu'être porteur des mêmes droits que ces concitoyens et être reconnu dans ses droits est conditionné, d'une part, par la connaissance des normes qui gèrent la vie sociale et par la conscience et le respect de ses propres devoirs vis-à-vis des membres de la communauté dont il fait partie ; d'autre part, 
par la reconnaissance de soi par d'autrui en tant que porteur de droits et de devoirs égaux. Honneth se réfère à Mead (1963) pour qui le concept d'« autrui généralisé" ", instance de normalisation et de contrôle, permet la reconnaissance réciproque. L'une des caractéristiques de la reconnaissance juridique réside dans sa portée universelle. Elle concerne l'homme indépendamment des spécificités. Dans ce sens, elle n'admet, comme le souligne Honneth en s'appuyant sur l'analyse de Marshall (1963), ni privilège ni exception, le statut ainsi que les différences ne rentrent pas en ligne de mire. Cette reconnaissance ne connaît pas de degrés (Honneth, 2010), car elle repose sur l'égalité des citoyens jouissant des mêmes droits.

\section{Le principe de solidarité et l'estime sociale de soi dans la sphère de la collectivité}

33 À l'inverse de la reconnaissance juridique qui ne tolère aucune particularité, car elle concerne le sujet en tant qu'être humain, la reconnaissance sociale prend en considération la valeur du sujet, ses capacités et sa singularité en tant que membre d'une communauté. Y vivre signifie participer à son maintien, à son développement et à son devenir. La valeur sociale des contributions individuelles au sein d'une communauté apporte à chacun de la reconnaissance et de la considération, éléments nécessaires pour élaborer un rapport positif à soi. Autrement dit, c'est la confirmation que les capacités individuelles, ayant contribué au bien-être de la communauté, ne sont pas dépourvues de valeurs aux yeux des autres membres de la communauté. En somme, ce qui entre en jeu ce n'est pas l'égalité des sujets, mais l'appréciation des valeurs et des qualités singulières qui aboutit à l'estime sociale de soi laquelle conduit à une solidarité entre les membres d'une collectivité (Honneth, 2010).

\section{Par ailleurs et comme le précise Honneth}

les rapports d'estime sociale sont, dans les sociétés modernes, l'enjeu d'une lutte permanente, dans laquelle les différents groupes s'efforcent sur le plan symbolique de valoriser les capacités liées à leur mode de vie particulier et de démontrer leur importance pour les fins communes. Il est vrai que l'issue - toujours provisoire - de telles luttes ne dépend pas seulement de la possibilité qu'ont les différents groupes d'accéder aux instruments du pouvoir symbolique, mais aussi de leur aptitude à orienter l'intérêt du public. (pp. 154-155).

En somme, la confiance en soi issue de l'amour et de l'amitié, le respect de soi comme signe d'égalité en droit ainsi que l'estime sociale de soi attestant de la valeur du sujet et de son utilité pour la communauté dont il fait partie, c'est cette conception de la reconnaissance qui permet à chaque sujet une existence humaine respectée.

\subsubsection{Raccrochage scolaire et définition commune de la situation}

Comme le précise Thomas (1984), «il y a toujours rivalité entre la définition qu'un individu produit spontanément d'une situation et celle que la société à laquelle il appartient met à sa disposition» (p. 80). Dans cette optique, le décrochage scolaire renvoie à une définition tensionnelle voire conflictuelle de la situation. L'élève ne perçoit plus l'école comme celle-ci se définit. Elle n'est plus vécue par l'élève comme un lieu d'épanouissement, d'ouverture, d'exploration. Le lien pédagogique devient tendu, les normes ne constituent plus une référence commune pour interagir et converger vers le même but. Quand ces tensions deviennent paroxystiques, on assiste à une rupture qui prend la forme de décrochage définitif.

37 En revanche, le raccrochage scolaire repose sur une définition négociée de la situation rendant possible l'implication de l'élève dans une dynamique d'apprentissage et 
permettant en particulier à l'enseignant de mieux accompagner l'élève pour atteindre un objectif commun, à savoir le raccrochage. La notion de définition de la situation nous semble pertinente pour cerner le sens que les acteurs en situation de coprésence (Goffman, 1974) confèrent à ce qui se passe dans leur «ici et maintenant». Toute situation se déroulant dans un cadre institutionnel repose sur une prédéfinition que l'institution met (impose) à la disposition des acteurs. Dans ce sens, cette prédéfinition constitue le contexte institutionnel et fait l'objet d'échanges et de négociations explicites ou implicites entre les différents acteurs de la situation. Dans le cadre du raccrochage scolaire, la prédéfinition de la situation, celle qui existe avant que les acteurs se rencontrent, tout ce que l'institution mis à leur disposition fonctionne comme un cadre facilitant le nécessaire travail de négociation entre enseignants et élèves. Cette négociation interroge à la fois la prédéfinition, les points de vue des acteurs, tentent de surmonter les obstacles et les freins rencontrés, et contribue à une coconstruction d'une définition commune de la situation susceptible de faire advenir le raccrochage. Cette définition partagée est l'un des signes exprimant une volonté réciproque pour travailler ensemble.

Une définition partagée entre acteurs permet de maintenir leurs liens et de mutualiser leurs efforts en vue d'atteindre leurs objectifs communs. Comme le précisent Lenoir et al. (2007), la situation est dynamique et évolutive « elle émerge de l'interactivité des sujets et de leur processus négocié de construction de sens » (p. 43). Dans cette perspective, elle est intersubjective et constitue une occasion pour interroger le sens des savoirs, le sens de l'école. Il s'agit en effet, ainsi que des auteurs comme Charlot, 1997, 1999 ; Glasman, 2004, 2002 ; Dubet, 2014 ; Blaya, 2010 l'ont à plusieurs reprises évoqué, d'un problème central.

Par ailleurs, le raccrochage scolaire demeure vulnérable, car il ne corrige pas les inégalités scolaires et encore moins les inégalités sociales. Sa force réside dans la capacité et la volonté de différents acteurs y compris les élèves en situation de décrochage scolaire à travailler ensemble pour que le décrochage provisoire ne se transforme pas en déscolarisation, pour renforcer l'envie d'aller à l'école et pour que le savoir ait un sens pour l'élève. Il est important, ainsi que l'explique Dubet (2014), que

la vie scolaire et les apprentissages donnent aux élèves le sentiment de grandir, d'être plus intelligent et plus autonome. Je ne peux apprendre les mathématiques ou une langue étrangère seulement parce que c'est utile et obligatoire. Il faudrait aussi que j'y trouve un intérêt intellectuel. D'ailleurs, sans cet intérêt intellectuel « gratuit » je ne parviendrais pas à apprendre. Pour travailler à l'école, il faut que la culture scolaire soit légitime, «enchantée et perçue comme libératrice et «gratuite ». (p. 14)

\section{Démarche mobilisée}

En cohérence avec les questions qui ont servi de fil rouge à notre étude et vu l'approche retenue, nous avons opté pour une démarche ethnographique. Le recours à cette démarche est justifié également par une centration sur les points de vue des élèves en situation de décrochage scolaire et par la visée compréhensive qui oriente ce travail. Tenter de comprendre de l'intérieur le sens que les élèves confèrent à leur ici et maintenant et à leur devenir scolaire passe inéluctablement par le choix d'une «bonne distance épistémique» (Le Grand, 1989) qui est finalement une distance dynamique marquée par une variabilité du degré d'implication. L'acte de comprendre dans l'ethnographie interactionniste passe par une activité de dépliage et par une explicitation 
plutôt qu'une explication (Herman, 1993) des points de vue des acteurs. Dans cette perspective, l'ethnographie interactionniste s'inscrit pleinement dans le paradigme de la complexité telle que Morin $(1990,1999,2000)$ le définit. Notre démarche est composée de plusieurs étapes :

\section{Négociation d'accès au terrain}

Elle s'est faite progressivement et par participation aux différentes réunions organisées par le groupe de pilotage d'un projet intitulé «il faut tout un village pour éduquer un enfant ». Par ailleurs, cet accès a été largement facilité par la fonction ${ }^{3}$ qu'occupait l'un des auteurs de cette contribution au sein de cet établissement. La négociation avec les élèves et les parents d'élèves n'a pas rencontré de difficultés significatives d'autant plus que les parents sont concernés directement et impliqués dans le projet de raccrochage scolaire puis qu'ils participaient aux différents ateliers.

Il convient tout de même de préciser que l'ethnographie à laquelle nous nous référons ici s'inscrit dans le courant de l'interactionnisme symbolique en prenant en considération les différentes adaptations introduites par Lapassade (1997, 1998), Boumard (1994, 2005, 2011) ; et, avant eux, Woods (1990). Ce dernier précise que la démarche ethnographique

tient dans la place centrale qu'occupe la construction du sens chez l'individu, sa "définition de la situation ». Dérivée du travail de Mead et de l' «école de Chicago ", elle dresse une dichotomie entre le monde " naturel » qui existe hors de nous et au sein duquel nous sommes des organismes faits de pulsions et d'instincts et le monde «social », dans lequel le symbolisme (langage, gestualité) nous permet de donner un sens aux objets (Woods, 1990, p. 25).

\section{Composition de l'échantillon}

Comme nous l'avons précisé supra, notre échantillon initial est composé de trois catégories : enseignants, élèves et parents d'élèves. Cependant, nous nous limitons dans le cadre de cette contribution uniquement aux élèves en situation de décrochage scolaire ayant accepté, avec leurs parents, de participer aux différentes activités proposées dans le cadre du dispositif «il faut tout un village pour éduquer un enfant » et ayant été interviewés avant et pendant la fréquentation du dispositif. Au total, 12 élèves de $4^{\mathrm{e}}$ ( 5 filles et 7 garçons) tous issus de milieux défavorisés et dont certains parmi eux vivent des situations familiales assez difficiles ont participé à cette étude. Les collégiens sont tous en $4^{\mathrm{e}}$ au moment de l'étude et ils sont répartis dans cinq classes différentes. Au cours des six mois qui séparent le premier et le deuxième entretien, la fréquentation du dispositif atteint une moyenne de 78,5\%. Des renseignements relatifs à leur parcours scolaire ont été mis à notre disposition par la direction de l'établissement.

\begin{tabular}{|l|l|l|l|l|}
\hline $\begin{array}{l}\text { Code } \\
\text { élève }\end{array}$ & Age & sexe & Niveau de classe & $\begin{array}{l}\text { Fréquentation } \\
\text { dispositif (\%) }\end{array}$ \\
\hline E1 & 14 & F & $4 \mathrm{e}$ & 100 \\
\hline E2 & 15 & M & $4^{\mathrm{e}}$ & 71,4 \\
\hline E3 & 14 & F & $4^{\mathrm{e}}$ & 71,4 \\
\hline E4 & 15 & M & $4^{\mathrm{e}}$ & 85,7 \\
\hline E5 & 14 & F & $4^{\mathrm{e}}$ & 85,7 \\
\hline E6 & 14 & F & $4^{\mathrm{e}}$ & 71,4 \\
\hline
\end{tabular}




\begin{tabular}{|l|l|l|l|l|}
\hline E7 & 14 & $\mathrm{M}$ & $4^{\mathrm{e}}$ & 85,7 \\
\hline E8 & 14 & $\mathrm{M}$ & $4^{\mathrm{e}}$ & 85,7 \\
\hline E9 & 15 & $\mathrm{M}$ & $4^{\mathrm{e}}$ & 100 \\
\hline E10 & 14 & $\mathrm{M}$ & $4^{\mathrm{e}}$ & 71,4 \\
\hline E11 & 14 & $\mathrm{M}$ & $4^{\mathrm{e}}$ & 42,9 \\
\hline E12 & 15 & $\mathrm{~F}$ & $4^{\mathrm{e}}$ & 71,4 \\
\hline & $\begin{array}{l}\text { Moyenne } \\
\text { d'âge }: 14,3\end{array}$ & $\begin{array}{l}41,7 \% \\
7 \quad \text { garçons } \\
58,3 \%\end{array}$ & $\begin{array}{l}\text { Tous en } 4^{\mathrm{e}} \text { issus de } 5 \\
\text { classes différentes }\end{array}$ & $\begin{array}{l}\text { Taux } \\
\text { présence }: 78,5 \% *\end{array}$ \\
\hline
\end{tabular}

*Taux de présence calculé sur un ensemble de 7 séances proposées par l'intervenant du dispositif de mars à juin 2015

\section{Lieu d'investigation, genèse et présentation du projet et du dispositif}

Le collège sur lequel repose cette étude, fait partie de l'éducation prioritaire depuis de nombreuses années (aujourd'hui REP+). Ses effectifs sont stables depuis plusieurs années, environ 450 élèves qui proviennent pour une grande partie des quartiers inscrits dans la géographie prioritaire de la politique de la Ville. La caractérisation sociologique des parents des élèves est cohérente avec cette situation avec 71,5\% d'ouvriers et d'inactifs ${ }^{4}$ et conséquemment, un taux de $74,9 \%$ de boursiers (vs $60,2 \%$ dans l'académie de La Réunion). Par ailleurs, le taux de réussite au diplôme national du brevet est plus faible que le taux académique ou national, mais il est en constante évolution depuis près de cinq ans (+ 21 points). L'analyse des redoublements des élèves sortant du collège (aussi bien dans les filières générales que professionnelles) indique des taux bien plus élevés que les taux académiques, avec une présomption forte d'abandon scolaire. Pour terminer, le climat scolaire fait également partie des priorités de l'équipe du collège, dont un travail important et régulier avec les familles depuis plusieurs années. Le projet dont il est question dans cet article, a fait l'objet d'un diagnostic partagé avec un ensemble d'acteurs de la communauté éducative (principal et principal adjoint, conseillère principale d'éducation, conseillère d'orientation psychologue, assistante sociale, infirmières, coordonnateurs de niveau, animateurs des groupes de parole) permettant ainsi de relever un certain nombre de constats parmi lesquels: un absentéisme important, des retards fréquents, un climat scolaire tendu (entre élèves et entre élèves et adultes), une motivation faible pour les apprentissages scolaires, une mauvaise réputation de l'établissement, une faible estime de soi (à l'entrée au collège et/ou au fil de la scolarité au collège), une difficulté à mettre en place des actions pour impliquer durablement les parents, un besoin de formation des personnels de l'établissement, à la relation avec les élèves, les parents pour prévenir, gérer les conflits, une nécessité d'agir dans la durée, dans une perspective de continuité, dans une logique préventive.

De ce diagnostic partagé, un dispositif a été conçu et mis en œuvre par le collège qui l'a inscrit dans deux axes du projet d'établissement : 1- améliorer le climat scolaire et l'image de l'établissement; 2- améliorer la préparation à la poursuite d'étude au lycée (en anticipant le décrochage des élèves au lycée). Ce projet de raccrochage scolaire a pour objectifs principaux de favoriser la persévérance scolaire, réduire l'absentéisme, motiver 
les élèves dans les apprentissages scolaires, et favoriser un meilleur rapport à l'école en développant l'estime de soi et en valorisant les compétences des élèves. L'originalité du dispositif réside dans le fait qu'il propose des actions à une pluralité d'acteurs : les élèves, les parents et les personnels de l'établissement. Ainsi, s'inscrit-il dans un projet global de réussite éducative visant à construire des partenariats au niveau du territoire.

Le public cible du projet «il faut tout un village pour éduquer un enfant », est le niveau quatrième. 19 élèves font partie du projet en début d'année 2015, leur suivi est prévu sur deux ans (2014-15 et 2015-16), leur recrutement s'est fait en utilisant le questionnaire de Blaya $^{5}$ afin de dépister les élèves, définir leurs profils et la nature des interventions la plus appropriée. A ces résultats, se sont ajoutées les observations des différents acteurs de l'établissement. Le "recrutement" des élèves s'est fait sur la base du volontariat et l'inscription des parents à l'action de soutien à la parentalité de ce projet a été un préalable indispensable. À la mi-février 2015, 19 élèves et 12 parents avaient accepté de participer au projet. 1 élève s'est désisté dès la première séance. 1 élève ne s'est jamais présentée, toujours absente du collège.

Le dispositif conduit à une série d'actions en direction des élèves (6 séances animées par une spécialiste de l'éducation pour la santé de mars à juin 2015 puis des séances prévues sur l'année scolaire 2015-16, à raison de 6 à 7 par semestre). Ce qui fait le caractère inédit de cette expérience, c'est la mise en place d'une série d'actions en direction des parents de ces élèves ( 6 séances animées par la même professionnelle et le même jour que les séances des élèves), mais également, en parallèle, des actions de formation en direction des personnels de l'établissement sous la forme d'un Plan de Formation Établissement (PFE), organisé et animé par la même professionnelle, auprès du personnel volontaire (15 personnes) afin d'accroître la qualité de la relation pédagogique et mieux gérer les conflits, voire les prévenir.

Dernier aspect du dispositif, l'articulation des actions proposées par l'établissement avec celles qui sont proposées par le Contrat Urbain de Cohésion Sociale (CUCS) et animées par des adultes relais, dont une Opération Ville/Vie/Vacances (OVVV). Ces précisions relatives à l'environnement scolaire (et celles déjà évoquées) facilitent le nécessaire travail de contextualisation. Enfin, cet établissement a l'avantage de nous offrir la possibilité de suivre le passage d'une situation de décrochage vers celle de raccrochage scolaire via le dispositif mis en place.

\section{Recueil et analyse des données} effectué en deux temps 24 entretiens répartis de la manière suivante :

12 entretiens réalisés avant que les élèves de notre échantillon ne s'engagent dans le dispositif de raccrochage scolaire. L'objectif étant de saisir la perception que le sujet a de lui-même avant de fréquenter le dispositif. Dans cette perspective, nous avons construit une grille d'entretien composée de rubriques favorisant l'expression des points de vue du sujet sur ses rapports à son histoire scolaire et au monde scolaire (voir annexe 1);

12 entretiens effectués avec les mêmes élèves qui ont fréquenté pendant quatre mois le dispositif « il faut tout un village pour éduquer un enfant». Le but de ce second recueil de données est de savoir comment ces élèves ont vécu cette expérience et de tenter de savoir si le dispositif a contribué à l'enclenchement d'un processus de raccrochage scolaire et de microtransformations dans les perceptions initiales identifiées lors du premier entretien (voir annexe 2). 
L'ensemble des données recueillies a été traité selon la technique de l'analyse thématique. Ensuite, les résultats obtenus ont été comparés en vue de repérer quelques éléments saillants relatifs aux objectifs de départ.

\section{Analyse et interprétation des résultats}

Notre objectif est, rappelons-le, de comprendre, à travers les points de vue exprimés par 12 collégiens inscrits dans le dispositif «il faut tout un village pour éduquer un enfant ", le double processus de décrochage et de raccrochage scolaires. La démarche ethnographique choisie devrait faciliter la saisie du sens que les sujets confèrent à leur ici et maintenant, mettre en évidence des altérations et des microtransformations dans le rapport à soi, à l'école, aux enseignants, aux pairs et à la famille.

\subsection{Perception des difficultés scolaires - origine des difficultés - solutions envisagées}

Les points de vue exprimés par les collégiens permettent d'appréhender le sens que les sujets confèrent à l'origine de leurs difficultés scolaires, de quelle façon ils les perçoivent et les solutions qu'ils peuvent ou non envisager pour les faire évoluer.

La plupart des collégiens interviewés expriment des difficultés de compréhension: «j'arrive pas à comprendre les choses, c'est trop dur pour moi», et presque systématiquement, ces difficultés sont reliées à la matière enseignée, aux disciplines, dont les plus fréquemment cités sont: la technologie, la physique : "je comprends rien"; l'histoire-géographie: «je comprends, mais pas vraiment» et le domaine des mathématiques : «je comprends pas les calculs...». Les premières (Sciences et sciences humaines sociales) sont des disciplines scolaires centrées sur la conceptualisation, elles nécessitent de faire appel tout particulièrement à la fonction abstractive, à cette capacité à déconnecter les mots et le discours de la représentation concrète, à passer au générique, à créer et penser des catégories, à la capacité de mettre en relations, dont l'élève "décrocheur" ne se saisit que difficilement, ayant davantage recours à un fonctionnement "mécanique ", analogique, connu, et rassurant, même si la réussite n'est pas toujours là. Parmi les points de vue exprimés par les collégiens, la difficulté à comprendre et utiliser le français en situation scolaire est fréquemment évoquée : « Moi des fois, je comprends pas trop bien le français, les mots en français, l'orthographe aussi pas trop bien et parler en français ", "j'arrive pas vraiment à parler en français » ou encore « en français, c'est dur à apprendre ». Ces matières (langues et mathématiques), considérées comme apprentissages fondamentaux, qui permettent de communiquer les réalités, sont aussi celles pour lesquelles la société civile souhaiterait un recentrage. Discipline singulière, plurielle et transversale, objet d'une discipline et véhicule des autres, le français semble cristalliser au sein de l'environnement social et scolaire de l'élève, ce qui rend difficile toute évolution : le poids du regard de la famille, de ses pairs et de l'enseignant le maintient, bien malgré lui, dans son rôle d'élève en difficulté et se manifeste par des signes d'insécurité linguistique (Bretegnier, 2002).

61 Du fait de leurs origines, ces causes sont inconscientes chez le collégien et celles qu'il évoque, renvoient parfois à des problèmes d'ordre cognitif : «Ca vient du cerveau » ou plus fréquemment à un système de valeurs négatives : «c'est juste que j'apprends pas »; 
«des fois, c'est parce que j'apprends pas mes leçons, en fait, c'est pour ça »; « des fois, je comprends pas parce que j'écoute pas », faisant émerger ici la question de l'amour et de la confiance en soi : «je suis pas trop fort à ça c'est tout (en parlant de ses difficultés en calcul), et de l'estime sociale de soi : «j'ose pas demander en fait, j'ose pas dire, parce qu'il y a certains profs qui veulent pas répéter les questions »; «les professeurs sont très sévères, je ne comprends pas les choses après ». Aucun élève n'évoque la situation sociolinguistique de La Réunion, la langue créole n'est pas suggérée alors que certains s'entretiennent en créole avec l'enquêteur ou utilisent des formes interlectales (Souprayen-Cavery, 2010).

Dans la plupart des situations, l'expérience scolaire est vécue comme une souffrance, qui peut s'exprimer de manière explicite: «je vis ces difficultés, durement» ou indirectement: «j'essaie mais j'arrive pas»; «j'essaye de comprendre, mais quand j'arrive pas, bah je laisse un peu tomber ", conduisant à une spirale de l'échec et à un décrochage avéré. En outre, ainsi que l'expliquent à juste titre Dubet et Martuccelli (1996), «la peur de l'échec ne renvoie pas seulement à la crainte de compromettre l'avenir, elle est plus encore la crainte diffuse d'être conduit à se mépriser soi-même. Les élèves qui échouent s'enfoncent dans le silence, le retrait et le malheur, d'autres se retournent contre l'école afin de préserver leur propre identité » (p. 251).

Quand on les questionne sur des solutions possibles pour surmonter leurs difficultés, un seul collégien évoque le lien entre réussite scolaire et maîtrise de la langue française : « Je dis que pour surmonter mes difficultés, il faut que je prononce bien les mots, bien parler, l'orthographe, bien apprendre et bien écouter aussi ». Plus souvent, les collégiens se trouvent démunis devant cette question : «je sais pas », «j’ai aucune idée » ou évoquent les notions générales d'apprentissage et d'écoute. La question de la reconnaissance se trouve alors déguisée sous le terme de « comportement » renvoyant au rapport à autrui, rapport à l'enseignant, aux pairs, à la famille : «mon professeur de CM2 m'a humiliée un jour, cela ne m'a pas plu et depuis, je n'ai plus travaillé. Maman était venue me chercher, car j'étais malade et mon professeur lui a dit que je mentais. J'ai bien vu qu'il ne m'aimait pas et c'est pour cela que je n'ai plus travaillé depuis ${ }^{6}$. Ce propos tenu en créole par une collégienne (traduit ici en français) est l'expression d'un déni de reconnaissance, une forme de mépris social par élimination symbolique de l'Autre (Honneth, 2010 ; Lenoir et Froelich, 2012), qui conduit à une perte de dignité et d'estime sans laquelle il est difficile d'apprendre, voire même d'exister (Caillé, 2004).

\subsection{Perceptions des collégiens avant le démarrage du projet}

\subsubsection{Rapport à soi}

L'expérience négative du décrochage nous renseigne sur l'importance de la reconnaissance $\mathrm{du}$ sujet en tant que personne singulière. Parmi les collégiens questionnés, deux groupes se dégagent : ceux que nous dénommons les "perdus », qui signifient leur manque de repères sociaux, familiaux et/ou scolaires : «je galérais un peu et je me perdais » et les collégiens « ordinaires », ceux qui désirent avant tout ne pas se distinguer de leurs pairs : « je suis normal, normal comme tous les autres ». Les collégiens du premier groupe sont en recherche d'une singularité, le «je » est prépondérant et la forme de reconnaissance est de type "identification» alors que le second groupe, cherche davantage à être reconnu au sein du groupe de pairs, reconnu en tant qu'être 
humain, ayant même valeur et appartenant à la même catégorie sociale, celle des collégiens de $4^{\mathrm{e}}$ (affiliation à des alter ego, création d'une communauté).

$\mathrm{Au}$ cours de sa biographie scolaire, le collégien saisit de façon relativement lucide le point de rupture situé dans la majorité des cas, en deuxième année du collège : «En $6^{e}$, j'étais normal, comme tous les autres "; "J'apprenais tout le temps, j'étais... un bon élève "; «en $5^{\mathrm{e}}$, ça a commencé »; « en $6^{\mathrm{e}}$, j'avais une bonne moyenne et en $5^{\mathrm{e}}$, ça a commencé à diminuer». En quatrième, le "décrocheur " aspire à être d'autant plus reconnu qu'il «bascule» vers l'adolescence du fait du processus biologique de la puberté et d'une double révolution physiologique et psychique. L'adolescent a nécessairement à prendre en compte la transformation psychique qui en découle, qui se fait sereinement quand amour de soi, confiance en soi et estime sociale de soi sont présents. Pour l'adolescent «fragile », une friction supplémentaire s'imposerait probablement à lui, le conduisant vers un décrochage certain.

\subsubsection{Rapport à l'école et à l'enseignant}

L'analyse des réponses met en évidence un rapport à l'école en dehors de soi. Une majorité de collégiens disent d'eux qu'ils notent les cours, font leurs devoirs, participent en classe et pour autant, ils n'hésitent pas à dire également qu'ils sont souvent absents, n'aiment pas l'école, et que parfois «c'est énervant ». Quelques-uns ont conscience de ne pas exercer leur métier d'élève pour reprendre le propos de Perrenoud (1994): «j'ai parfois du mal à rattraper les cours, à écrire »; « je vais à l'école le matin parce que mwin lé obligé (je suis obligée) »; « je suis absente parce que des fois, j'ai pas envie ».

Pour accepter un temps l'idée d'absence de désir: "J'aime pas trop l'école », «j'aime pas", "parce que lé ennuyant», "parce que c'est nul», l'adolescent se centre sur ses pairs, sur cet autre qui lui ressemble: «j'ai envie d'y aller (à l'école) des fois pour voir mes camarades »; « La joie. Retrouver mes camarades ». Chacun veut être reconnu par les autres (au sens de Hegel dans La Phénoménologie de l'Esprit publiée en 1807) et a «le désir impérieux de se faire reconnaître comme conscience de soi » (Kojève, 1947). C'est par la médiation que l'adolescent peut accepter l'idée d'aller en cours, se montrant capable de sacrifier ses intérêts immédiats au profit du groupe, des pairs, par ce « désir d'autrui ", considéré "en tant que composante intrinsèque et constitutive du rapport d'objet, une donnée interne de sa structure, assure le détour nécessaire, surmonte et résout la rupture (comble la distance) que le sujet établit en produisant l'objet en extériorité par rapport à lui » (Lenoir, 1993, p. 68). On note des éléments contradictoires dans le discours des adolescents qui peuvent associer l'école à l'idée de bien être : «je me sens bien à l'école » et, dans le même temps, disent ne pas se sentir reconnus par leurs enseignants : «ils disent que des fois, je suis bien dans ma tête des fois je suis pas bien », " que je suis nul».

68 La question de la reconnaissance s'impose quand il s'agit d'évaluer la qualité de la relation pédagogique. L'unique sens du travail scolaire réside dans la recherche du « désir du désir de savoir de l'enseignant»(Lenoir, 1996, p. 240). Deux formes de déni de reconnaissance prédominent dans le discours des collégiens : l'invisibilité sociale : « il y en a qui ne savent même pas (qui je suis) », « je sais pas ce qu'ils pensent de moi », et le mépris social (cf. supra) «Pour eux (les professeurs), je suis bonne à rien», « ils disent que je suis pas concentré, que j'écoute pas et que j'ai beaucoup de difficultés », « qu'il faut 
que je me calme ». Les propos des collégiens rendent ici compte du lien sensible qui peut exister entre reconnaissance de soi et désir d'apprendre.

\subsubsection{Rapport aux pairs} d'appartenance dont les adolescents en crise ont un besoin impérieux. Presque tous les collégiens interrogés déclarent avoir un ou des groupes d'amis, à l'école et hors l'école. $\mathrm{Au}$ sein du groupe, le collégien peut jouer le rôle de meneur, le "chef », celui qui met l'ambiance, celui qui est singulier (le plus petit du groupe)... Le jeune collégien dont le rapport à l'école est fragilisé, parvient à se rassurer, se protéger, exister, s'affirmer ainsi dans son rapport aux pairs. Cette phase de déconstruction ordinaire peut prendre parfois la direction du décrochage scolaire quand la reconnaissance figure au rang des absents : "quand on pose une question, ils (les professeurs) disent: Ah non, j'ai déjà répété donc débrouillez-vous ». Prenant appui sur la théorie de la régulation sociale (Hirschi, 1969) qui accorde une attention particulière à l'influence des pairs, les propos des collégiens indiquent qu'ils adoptent certains comportements transgressifs parce que ces comportements ont été montrés, encouragés et renforcés par leurs groupes de pairs : « en $5^{\mathrm{e}}$, c'est là que j'ai commencé à ravager (perturber la classe, le cours)... C'est l'entraînement avec les amis... Je suis en train de me dégrader » Le degré de pression des pairs et/ou d'attachement à ces pairs est sans doute prédictif des risques de transformation du décrochage provisoire en un décrochage scolaire avéré.

\subsubsection{Rapport à la famille}

Si la théorie de l'apprentissage social reconnait l'importance des influences prosociales de la famille et de l'école, les propos des collégiens pré-décrocheurs font apparaître un lien étroit entre degré d'implication parentale et reconnaissance selon un double processus entre le singulier et l'universalité : un suivi irrégulier des notes et de l'emploi du temps (rôle assigné plutôt au père), la venue au collège quand la famille est convoquée (part attribuée plutôt à la mère), la présence rare d'adjuvants pour les devoirs (grand frère ou grande sœur)... Dans le cas des familles populaires qui constituent notre échantillon, on retrouve la théorie de la reproduction sociale chère à Bourdieu (1970) : «Ils disent (mes parents) qu'ils ont pas réussi à l'école et qu'ils veulent pas que je suive le même chemin qu'eux »; « ma maman elle me dit de faire mes devoirs et de me préparer vite pour aller à l'école ». Peu de place au dialogue entre parents et adolescent à propos de leur propre histoire scolaire ou de la vie de collégien de leur enfant. Avoir des mots pour le dire au sens de Bourdieu (1982), c'est d'une certaine manière s'approprier les codes scolaires et avancer d'un pas dans la construction identitaire de leur enfant-élève.

\subsection{Des microtransformations, révélatrices de raccrochage}

71 Après quatre mois de fréquentation du dispositif « Il faut tout un village pour éduquer un enfant», un processus de raccrochage scolaire s'enclenche rendu visible par des microchangements aussi bien dans le rapport à l'école et aux enseignants que dans le rapport à soi et à autrui. La perception de soi par soi évolue : un rapport positif à soimême dans l'ici et maintenant qui conduit à une plus grande confiance en soi : «je me croyais plus méchante, mais je me suis trouvée plus gentille au fond de moi, je me vois 
plus grande et plus responsable »; " avant, je perturbais et je criais Monsieur ! et là, non, je travaille bien, je pense que mes profs me voient comme un enfant intelligent, calme. ». Le rapport à l'autre parait transformé sous le filtre d'une reconnaissance réciproque : «Ce qui a changé, c'est que je parle, je me sens bien dans la classe, les cours, en dehors... aller vers les autres, parler avec tout le monde... tandis qu'avant, mi parle juste avec deux trois (amis) ». Le rapport à l'école bénéficie de microtransformations multiples: 1- la coconstruction d'une définition commune de la situation de décrochage-raccrochage scolaires: "Avant mi té vois l'école comme si que c'était une obligation, fallait tout le temps apprendre, mais en fait, c'est pour nous même au final. Si nou veut pas réussir, nou réussira pas dans la vie plus tard. Même si ou gaign pas un grand nafèr, ou va gaign au mwin un bout »; 2- une estime sociale de soi traduite par une participation plus fréquente dans des matières comme les mathématiques et le français; 3- une reconnaissance sociale qui prend en considération la valeur du sujet, ses capacités : « il y a un professeur qui m'a dit que j'ai une note d'effort, parce que j'ai fait des efforts »; « je me sens mieux parce que les profs sont fiers de moi »; «les profs me voient comme un garçon intelligent et concentré dans son travail ».

En ce qui concerne les résultats scolaires, les collégiens notent parfois une progression importante : « Oui, ma gaign un 20 en technologie (j'ai obtenu)»; « 18 en français, 12 en maths »; la plus fréquente des progressions se situe en français et cela mériterait sans doute de s'y attarder (ce que nous ne ferons pas ici). Il semble aussi y avoir un impact sur les procédures d'apprentissage : "parce qu'avant je ne travaillais pas. Je ne comprenais pas, il y avait personne pour m'aider, mais là, il y a mon ami qui m'aide un petit peu. Il me donne pas les réponses, mais il essaie de me faire comprendre la leçon ».

Le rapport à la famille, paraît au contraire peu touché, avec un lien jugé opaque par les collégiens entre les attitudes de leurs parents vis-à-vis d'eux-mêmes et le dispositif auquel les jeunes participent: «je sais pas si ça a changé ou pas»; "euh (gêne), pas forcément». On ne constate que peu d'effets sur les attitudes des parents: "ils m'écoutent mieux qu'avant »; «elle prend plus soin de moi qu'avant ». La situation est complexe et le lien école-famille sera à questionner dans une prochaine étude par le biais d'une analyse thématique du discours des parents.

Pour revenir sur le dispositif et ses effets sur l'engagement des collégiens à moyen terme, on notera d'abord un taux de présence assez stable entre les 7 séances qui ont eu lieu entre mars et juin de leur année de $4^{\mathrm{e}}(78,5 \%)$ et les 11 séances de l'année suivante au moment où ces jeunes sont en classe de $3^{\mathrm{e}}(75 \%)^{7}$. L'engagement de ces collégiens dans le dispositif « il faut tout un village pour éduquer un enfant » semble également modifier de manière sensible leurs perceptions initiales et enclencher un processus de raccrochage scolaire. Les raisons sont sans doute plurielles, mais nous choisissons de nous attarder sur le dispositif mis en place dans ce qu'il propose aux jeunes sous des formes scolaires originales (groupes de parole, actions de coaching, séances visant à la restauration de l'estime de soi (connaissance de soi, capacité à s'exprimer et à parler de soi...), à la valorisation de partenariats et à la cohésion du groupe.

De nombreux jeunes posent en effet un regard positif sur ce dispositif inédit : « moi j’aime bien »; «c'est bien pour aider l'autre en difficulté »; «j'aimerais continuer encore car m'apporte des choses, par exemple, que dans la vie, tout n'est pas pareil»; «il fait avancer les élèves, il fait rêver les élèves, il met la pression aussi, mais il fait avancer ». Ainsi, pour reprendre les propos de Francequin (2009) dans son hommage à Lévine, il faudrait retenir du dispositif deux bases fondamentales : 
une conviction, celle que l'enseignant (l'animateur, le formateur...), en tant que responsable des conditions d'apprentissage, doit se doter de trois capacités essentielles: "la capacité d'alliance, la capacité de réalisme, la capacité de confiance dans l'aventure humaine. Elles font partie de tout accompagnement sécurisant de l'intérieur, qui par une présence qui pose «je fais face à la vie et j'entends vos craintes » peut rassurer l'autre avec réalisme. Le réalisme, c'est amener la personne enfant, adolescent ou jeune adulte à distinguer ce qui se fait de ce qui ne se fait pas, à distinguer l'essentiel de ce qui ne l'est pas » (p. 572). souligne Lévine (2000), permettrait de former des liens de filiation qui relèvent du « réparable ». Le « regard photo » serait celui qui fige, qui referme la temporalité sur les difficultés. Le "regard cinéma", inscrirait l'autre dans une temporalité ré-ouverte, il donne à intérioriser que, malgré toutes les difficultés, il est possible de se vivre comme porteur du passé, porteur de son présent, mais aussi porteur d'une trajectoire qui situe et relativise passé et présent, pour que la vie puisse continuer. Enfin, les perceptions des collégiens sont en adéquation avec les propos de Winnicott (1975) : le passage de la perte du sentiment d'exister au « sentiment que la vie vaut la peine d'être vécue » (p. 91).

\section{Conclusion}

À l'issue de cette recherche qui visait à percevoir et à concevoir de façon interreliée le décrochage provisoire et le raccroche scolaire, nous sommes parvenus à mettre en exergue les points de vue des élèves ainsi que les microtransformations concernant le rapport de ces élèves à l'école, à l'enseignant et à eux-mêmes.

Il nous a semblé important de nous focaliser sur le décrochage provisoire, qui demeure peu étudié en comparaison avec la déscolarisation et de l'abandon scolaire. En tant qu'entre-deux, le décrochage provisoire reste le lieu de toutes les possibilités. Nous avons ciblé l'une d'entre elles: celle du raccrochage scolaire que nous avons tenté d'appréhender à partir du dispositif « Il faut tout un village pour éduquer un enfant ».

À la différence des autres dispositifs qui se situent en dehors de l'établissement et qui n'impliquent pas de manière active les différents acteurs concernés de près ou de loin du décrochage scolaire, le dispositif «il faut tout un village pour éduquer un enfant " a l'avantage de montrer que le décrochage n'est pas l'affaire d'un élève isolé, mais celle de toute la communauté éducative. Cette préoccupation collective est de nature à faire cesser le mépris, la stigmatisation au profit d'une reconnaissance considérée comme une condition sine qua non de toute tentative de raccrochage scolaire.

Par ailleurs, le fait que ce dispositif est situé au sein de l'établissement scolaire, assurant une continuité spatiale, ne provoque pas chez l'élève le sentiment d'être à part et ailleurs. De la même façon, l'organisation des activités au sein de ce dispositif ne constitue pas une rupture au niveau du temps scolaire. Au-delà cette double continuité, un des points forts de ce dispositif réside dans le fait de ne pas penser le traitement du décrochage scolaire sur le mode de la simple prise en charge et de la focalisation sur les difficultés de l'élève.

81 La philosophie qui sous-tend ce dispositif accorde une place singulière à la conscientisation, qui n'est une forme de moralisation, comme une façon d'entrer dans le processus de raccrochage scolaire. En remplaçant l'imposition par la proposition et le déni de reconnaissance par la reconnaissance de l'autre en tant être singulier, égal en dignité et membre d'une communauté au sein de laquelle il jouit des droits et remplit des

Questions Vives, Nº 25 | 2016 
devoirs, ce dispositif place l'élève dans des conditions optimums pour rendre le raccrochage scolaire possible.

On retiendra de ce dispositif l'enseignement suivant: c'est, en partie, en termes d'implication collective et d'accompagnement qui fait advenir l'autonomie de l'élève, la confiance en soi et l'estime sociale de soi qu'il conviendrait de penser la lutte contre le décrochage scolaire. Les prises en charge unidimensionnelles et décontextualisées sont davantage des luttes contre les décrocheurs plutôt que contre le décrochage.

Pour terminer, cette recherche, qui loin d'être achevée, ouvre sur plusieurs perspectives. La complexité du phénomène du décrochage exige de construire une vision globale afin que le traitement ne soit pas morcelé et centré sur l'une des composantes de ce phénomène. C'est dans cette optique que nous avons songé poursuivre notre recherche en appréhendant les points de vue des parents, qui sont d'ailleurs impliqués dans ce dispositif, et en examinant l'effet de leur participation sur le rapport parent-enfant, le rapport à l'école...

Par ailleurs, si le rapport des élèves à l'enseignant a subi quelques changements qui ont renforcé le lien pédagogique, il serait pertinent, dans la continuité de cette recherche, de savoir si le rapport des enseignants aux élèves, perçus initialement comme des "décrocheurs sur place ", a changé suite au passage de ces derniers par le dispositif de raccrochage scolaire.

Dans la même veine, des investigations pourraient être menées afin de savoir si des enseignants, à l'issue de cette expérience concluante de raccrochage scolaire, ont revisité leur manière de faire classe, ont fait d'autres choix pédagogiques, ont engagé des activités différentes en vue de prévenir le décrochage scolaire et d'améliorer sensiblement la qualité du lien pédagogique.

Cette transversalité de notre recherche, qui reste à construire, ouvre sur une perspective comparative qui ne se limiterait pas uniquement à identifier les similitudes et les différences entre les visions de différents acteurs de la communauté éducative, mais qui déboucherait sur une identification et analyse des freins à un dialogue constructif et sur des initiatives de dépassement de ces obstacles.

Dans le contexte réunionnais, cette recherche invite à une analyse critique de la manière dont les dispositifs locaux engagent des actions en faveur des décrocheurs.

\section{Annexe 1 - Grille d'entretien élève Phase $n^{\circ} 1$}

\section{Partie 1 : Avant l'engagement dans le projet}

1) Comment l'élève se voit, la perception de soi

Peux-tu me parler de toi en tant qu'élève ?

Comment te décris-tu en classe? Dans la cour?

Comment étais-tu en 6e ? 5e ?

Selon toi, est-ce que cela a évolué ?

Si oui, comment tu trouves cette évolution?

As-tu déjà redoublé ?

As-tu déjà été absent? 
Si oui, pour quelle raison?

Est-ce que tu participes activement en classe (lever le doigt/ interrogé) ? Notes-tu les cours? Fais-tu tes devoirs?

2) Comment l'élève perçoit l'école

Que penses-tu de l'école ? Pourquoi ?

Qu'est-ce qui te pousse à aller tous les matins à l'école?

Est-ce que tu as envie d'y aller?

Comment tu te sens à l'école?

3) Comment il est perçu par les autres (pairs / enseignants) // Comment l'élève qualifie sa relation (humaine) avec les pairs // qualification du lien pédagogique

Est-ce tu as des copains à l'école?

Et en dehors de l'école?

Fais-tu partie d'un groupe?

Si oui, quelle est ta position dans ce groupe?

Que pensent tes copains de toi?

Est-ce que tes difficultés scolaires font l'objet de moqueries par d'autres élèves?

Si oui, comment as-tu réagi à leurs moqueries?

Est-ce que tu en as parlé à tes professeurs, à tes parents... ?

Qu'est-ce que les professeurs disent de toi?

Te sens-tu apprécié par tes professeurs?

Si oui, quels sont les signes qui te le montrent?

Penses-tu que cette reconnaissance est capitale pour ta réussite scolaire ? est-ce que c'est important pour toi?

Sinon, pourquoi?

Comment perçois-tu ta relation avec l'ensemble de l'équipe éducative? (CPE ? Assistant d'éducation? Documentaliste ? Principal ? Principal Adjoint ?) A qui t'adresses-tu le plus facilement?

4) Qualification des attitudes des parents

Est-ce que tes parents (ou quelqu'un de ta famille/responsable légal) s'impliquent dans ta scolarité ?

Est-ce qu'ils vérifient tes absences ? Connaissent-ils ton emploi du temps? Regardent-ils ton agenda?

Est-ce qu'ils t'aident à faire tes devoirs? Viennent-ils aux réunions / convocations / invitations à des manifestations proposées par le collège?

Est-ce que tes parents te soutiennent dans ta scolarité?

Ont-ils été à l'école?

Est-ce qu'ils te parlent de leur scolarité à eux ?

5) Perception des difficultés scolaires // Origine des difficultés

Peux-tu me parler de tes difficultés scolaires?

D'où viennent-elles? Quelles en sont les raisons? 
Comment tu vis ces difficultés?

Prends-tu le temps de travailler les cours?

As-tu accès à Internet? Un endroit pour travailler? Travailles-tu en permanence? Au CDI?

Participes-tu à des heures de soutiens scolaires? Au collège ? Dans une association?

Rencontres-tu d'autres difficultés que celles que tu viens d'évoquer? (comportement...)

6) Solutions envisagées

Selon toi, comment surmonter ces difficultés?

Penses-tu qu'un changement de comportement aurait pu améliorer la situation ?

Qu'aurais-tu fait sans le projet de raccrochage?

\section{Partie 2 : Juste après l'engagement dans le projet}

7) Les raisons de l'engagement de l'élève dans le projet de lutte contre le décrochage scolaire

Pourquoi participes-tu à ce projet ?

Comment as-tu été informé?

Trouves-tu cela intéressant?

Peux-tu nous parler de ce projet?

Qu'est-ce qui t'a plu?

8) Les attentes

Qu'attends-tu de ce projet?

Penses-tu que cela va influencer la suite de ta scolarité?

Si oui, de quelle manière et dans quel sens?

Quelles sont aujourd'hui tes perspectives d'avenir?

Penses-tu que ce projet pourrait te « réconcilier » avec l'école?

9) Les réactions de : parents, pairs, enseignants

Comment tes parents (responsable légal) ont réagi à ton adhésion au projet?

Étaient-ils d'accord?

Pensaient-ils que tu accepterais?

T'ont-ils incité à participer au projet?

Comment tes amis/ copains de classes ont-ils réagi à ton adhésion au projet?

Que t'ont-ils dit?

T'ont-ils incité à participer au projet ?

Comment tes enseignants ont réagi à ton adhésion au projet?

T'ont-ils incité à participer ?

Que t'ont-ils dit?

10) Perception de soi juste après l'engagement 


\section{Annexe 2 - Grille d'entretien élève Phase $n^{\circ} 2$}

\section{I) Appréciations générales}

Est-ce que l'engagement dans ce projet a un peu changé l'image que tu avais de toi-même avant?

Si oui, dans quel sens?

Est-ce cette participation te valorise?

Penses-tu que ce projet te sera utile?

11) Perception de l'école

Est-ce que ton engagement dans ce projet a changé l'idée que tu avais de l'école ?

Si oui, dans quel sens?

Depuis ton engagement, est-ce que tu viens à l'école par plaisir ou par obligation?

Depuis ta participation, comment tu trouves les cours de tes professeurs?

À quoi servent-ils ?

Pour conclure, pourrais-tu me dire ce qui a changé (ou pas changé) depuis ton engagement dans ce projet?

\section{Comment ça va depuis notre dernier entretien?}

Comment trouves-tu les séances d'accompagnement scolaire?

Qu'est-ce que tu aimes dans ces séances? (obtenir des informations en lien avec: la relation enseignant-élève, la taille du groupe, la possibilité de participer, les contenus enseignés, la (les) manière (s) de l' (les) enseigner...)

Qu'est-ce qui te déplaît dans ces séances? (idem)

Est-ce que ces séances sont différentes de celles qui sont faites par tes professeurs?

Si oui, demandez des précisions

As-tu constaté des ressemblances entre ces séances (dispositif, classe d'origine) ?

Si oui, demandez des précisions

\section{II) Changements, progressions...}

Depuis que tu as commencé à fréquenter le dispositif «il faut tout un village pour éduquer un enfant » as-tu constaté des changements?

Perception de soi par soi

Est-ce que la participation au dispositif a valorisé l'image que tu avais de toi-même?

Si oui, pourquoi?

Si non, pourquoi?

Est-ce que tu as une meilleure image de toi ? Comment te vois-tu aujourd'hui ? (demandez toujours des précisions)

Aujourd'hui, tu te sens comment?

Est-ce que ce changement t'a aidé et t'aide à progresser scolairement ? 


\section{III) Retour sur le dispositif, l'expérience...}

211 Pour terminer, peux-tu me donner ton avis sur :

- le dispositif

- ton expérience 


\section{BIBLIOGRAPHIE}

Alaoui, D. (2016). Déni de reconnaissance et lutte pour la reconnaissance en milieu scolaire réunionnais. In Y. Lenoir, A. Froelich \& V. Zúñiga (dir.), La reconnaissance à l'école : quel impératif? Quels problèmes, enjeux et défis? Perspectives internationales (pp.129-157). Québec : Presses de l'Université Laval.

Ballion, R. (1995). La démobilisation scolaire. Conférence au collège Saint-Exupéry, Vélizy, le 5 avril 1995, ronéotypé MAFPEN de l'académie de Versailles.

Blaya, C. (2010). Décrochages scolaires, l'école en difficulté. Bruxelles : De Boeck (Pédagogies en développement).

Boumard, P. (2005). Le terrain comme écran. Réflexions sur une évidence trompeuse dans les sciences anthropo-sociales. Revue Européenne d'Ethnographie de l'Éducation, 4, 27-40.

Boumard, P. (2011). Des ethnologues à l'école. Paris : Éditions Téraèdre.

Boumard, P. (1994). Microsociologie du chahut. Pratiques de formation, 28, 163-169.

Bourdieu, P., \& Passeron, J.C. (1970). La reproduction sociale. : éléments d'une théorie du système d'enseignement, Paris : éditions de Minuit.

Bourdieu, P. \& Champagne, P. (1992). Les exclus de l'intérieur. Actes de la recherche en sciences sociales, 91, 71-75.

Bretegnier, A. (2002). Regards sur l'insécurité linguistique. In A. Bretegnier et G. Ledegen (éds.) Sécurité-insécurité linguistique : terrains et approches diversifiés, propositions théoriques et méthodologiques (pp. 7- 33). Paris : L'Harmattan.

Broccolichi, S. (2000). Désagrégation des liens pédagogiques et situations de ruptures. V.E.I.Enjeux, 122 .

Caillé, A. (2004). Présentation, Revue du MAUSS, 23, 5-28.

Charlot, B. (1997). Du rapport au savoir, éléments pour une théorie. Paris : Anthropos.

Charlot, B. (1999). Le rapport au savoir en milieu populaire. Paris : Anthropos.

De Queiroz, J.-M. \& Ziolkovski M. (1994). L’interactionnisme Symbolique. Rennes : PUR.

Dubet, F. (2014). Préface. In T. Berthet et J. Zaffran (Dir), Le décrochage scolaire. Enjeux, acteurs et politiques de lutte contre la déscolarisation (pp. 9-17). Rennes : PUR.

Dubet, F. \& Martuccelli, D. (1996). A l'école. Sociologie de l'expérience scolaire. Paris : Seuil.

Francequin, G. (2009). Hommage à Jacques Lévine. L'orientation scolaire et professionnelle, 38(4), 567-572.

Glasman, D. (2000). Le décrochage scolaire : une question sociale et institutionnelle. VEI enjeux, $122,10-25$.

Glasman, D., \& Oeuvrard, F. (2004). La déscolarisation. Paris : La Dispute.

Glasman, D. (2014). Le décrochage scolaire comme processus. In T. Berthet et J. Zaffran (Dir), Le décrochage scolaire. Enjeux, acteurs et politiques de lutte contre la déscolarisation (pp. 31-42). Rennes : PUR. 
Herman, J. (1994). Les langages de la sociologie. Paris : PUF.

Goffman, E. (1974). Les rites d'interaction. Paris : Ed. de Minuit.

Honneth, A. (2004). La théorie de la reconnaissance : une esquisse. Revue du MAUSS, Dossier De la reconnaissance - Don, identité et estime de soi, 23(1), 133-136.

Honneth, A. (2006). La société du mépris. Paris : La Découverte.

Honneth, A. (2010). La lutte pour la reconnaissance. Paris : Éditions du Cerf (1 $1^{\text {re }}$ éd., 1992).

Hirschi, T. (1969). Causes of delinquency. Berkeley : University of California Press.

Kojève, A. (1947). Introduction à la lecture de Hegel. Leçons sur la phénoménologie de l'esprit professées de 1933 à 1939 à l'École des Hautes-études réunies et publiées par Raymond Queneau. Paris : Gallimard.

Lapassade, G. (1997). Les microsociologies, Paris : Anthropos.

Lapassade, G. (1998). Microsociologie de la vie scolaire, Paris : Anthropos.

Le Breton, D. (2004). L'interactionnisme symbolique. Paris : PUF.

Le Grand, L. (1989). La bonne distance épistémique n'existe pas. Education Permanente, n $100-101$, p. 109-122.

Lenoir, Y. (1993). Entre Hegel et Descartes : de quels sens peut-il être question en didactique ? In P. Jonnaert et Y. Lenoir (dir.), Sens des didactiques et didactique du sens (pp. 29-99). Sherbrooke : Éditions du CRP.

Lenoir, Y. (1996). Médiation cognitive et médiation didactique. In C. Raisky et M. Caillot (dir), Le didactique au-delà des didactiques. Débats autour de concepts fédérateurs (pp. 223-251). Bruxelles : De Boeck Université.

Lenoir, Y. (2009). En éducation, tout commence par la fin..., mais de quelle fin est-il question ? Le cas du Québec. Revue de l'Institut de sociologie, 1-4, 101-130.

Lenoir, Y., \& Froelich, A. (2012). La socialisation scolaire à l'aune des processus de reconnaissance dans les classes du primaire au Québec. Communication lors du colloque intitulé "La reconnaissance en éducation : une notion futile ou utile pour cerner les rapports sociaux ?", 17e Congrès de l'AMSEAMCE- WAER, Reims, 3-8 juin 2012.

Lenoir, Y., Maubant, P., \& Hasni, A. (2007). À la recherche d'un cadre conceptuel pour analyser les pratiques d'enseignement. Nouvelle série, $\mathrm{n}^{\circ} 2$, Sherbrooke : Documents du CRIE et de la CRCIE.

Lenoir, Y. (2012). « La socialisation entre éducation thérapeutique et lutte pour la reconnaissance : la question de l'estime de soi », dans Yves Lenoir et Frédéric Tupin (dir.), Les pratiques enseignantes entre instruire et socialiser (pp. 185-231). Québec : Presses de l'Université Laval.

Levine, J. \& Moll, J. (2000). Je est un autre. Paris : ESF

Morin, E. (1990). Introduction à la pensée complexe. Paris : ESF Éditeur.

Morin, E. (1999). La tête bien faite. Paris : Seuil.

Morin, E. (2000). Les sept savoirs nécessaires à l'éducation du futur. Paris : Seuil.

Perrenoud, P. (1994). Métier d'élève et sens du travail scolaire. Paris : ESF.

Rochex, J.-Y. (1998). Rapport au savoir, activité intellectuelle et élaboration de soi : du malentendu au décrochage? In M-C Bloch \& B. Gerde (coord.), Les lycéens décrocheurs. De l'impasse aux chemins de traverse (pp. 131-141). Lyon : Chronique sociale. 
Sarfati, F. (2013). Peut-on décrocher de l'université ? Retour sur la construction d'un problème social. Agora débats/jeunesses, 63, 7-21.

Souprayen-cavery, L. (2010). L'interlecte réunionnais, Approche sociolinguistique des pratiques et des représentations. Paris : L'harmattan.

Thomas, W. I. (1984). Définir la situation. In Y. Grafmeyer et Isaac Joseph (dir.), L'école de Chicago. Naissance de l'écologie urbaine (pp. 79-82), Paris : Aubier.

Winnicoot, D. (1975). Jeu et réalité, l'espace potentiel, Paris : Gallimard.

Woods, P. (1990). L'ethnographie de l'école. Paris : Armand colin.

\section{NOTES}

1. Selon Isaac Joseph (1998) un cadre est un « dispositif cognitif et pratique d'organisation de l'expérience sociale qui nous permet de comprendre ce qui nous arrive et d'y prendre part. Un cadre structure aussi bien la manière dont nous définissons et interprétons une situation que la façon dont nous nous engageons dans un cours d'actions » (p. 123).

2. «Pourquoi tous les élèves « démobilisés » ne « décrochent »-ils pas ? Est-ce uniquement parce qu'ils n'entrevoient pas d'issue ailleurs ? Ou bien est-ce pour rester dans ce qui, à certains égards, demeure un milieu protecteur ? Pour rester dans un réseau de camarades ? Ou, plus profondément, pour ne pas rompre avec ce qui, au sein du réseau familial et amical, demeure la « norme ", avec laquelle la rupture est lourde de risques et de coûts, en termes d'image de soi, de réinvention de rôle, ou de réattribution de place ? Est-ce pour avoir ses entrées dans un lieu où circulent et s'échangent des informations et des biens ? Ou pour attendre, tout simplement et magiquement, que la mobilisation « reparte " ?... Il pourrait ne pas y avoir de différence radicale entre ceux qui décrochent en s'esquivant du lycée et ceux qui, démobilisés, décrochent sur place; un continuum dans la population engagée, plus ou moins loin, dans un processus de démobilisation » (Glasman, 2000, p. 14).

3. L'auteure a été hébergée par l'établissement en question pendant quatre années durant sa mission de coordinatrice du réseau académique des centres de lecture-écriture de La Réunion. Pendant cette période, elle a mis ses compétences au service du collège en participant à la mise en place de projets pédagogiques de réussite éducative (PPRE) puis a été à l'initiative de la mise en place d'un livret d'accueil à destination des parents d'élèves de $6^{6}$. Elle a enfin été durant une année scolaire (2011-12), coordinatrice du dispositif «école des parents» implanté dans ce collège.

4. Données de l'année scolaire 2013-14

5. Il s'agit d'une adaptation du questionnaire d'évaluation du risque de décrochage scolaire en milieu scolaire (DEMS) du CTREQ (Potvin et al., 2003), au contexte français (Blaya, 2011). Ce questionnaire a pour objectif d'évaluer le risque du décrochage scolaire chez les élèves du secondaire (12 à 17 ans) et est complété par l'élève. Il se compose des cinq sous-échelles suivantes : Implication parentale (9 items), Attitudes envers l'école (9 items), Perception de son niveau de réussite scolaire (10 items), Supervision parentale (7 items) et Aspirations scolaires (4 items). Le questionnaire a été validé auprès d'un échantillon québécois (Potvin, Fortin \& Rousseau, 2009).

6. Mon professeur CM2 l'avait rabaisse a mwin et la pas plait pas mwin donc mi travaille pu. En fait, quand maman té vient chercher mwin, quand mi té malade bin li té dit n'importe quoi maman alé voir après té menteur et ou voit mi té aime pas donc ma pu travaille .

7. Un total de plus de 80 heures à raison d'une séance tous les 15 jours sur une période de six mois au cours de l'année de $4^{\mathrm{e}}$ et sur l'année scolaire de $3^{\mathrm{e}}$ 


\section{RÉSUMÉS}

Cette contribution traite $d u$ décrochage provisoire et $d u$ raccrochage scolaire d'élèves du secondaire. Notre objectif est de comprendre, à travers des points de vue exprimés par 12 collégiens, leurs perceptions d'une double expérience : celle du décrochage-raccrochage. Dans cette perspective, les données ont été recueillies à partir d'une démarche ethnographique facilitant la saisie du sens que les sujets confèrent à leur ici et maintenant. Elles ont été interprétées en référence à l'interactionnisme symbolique et à la théorie de la reconnaissance (Honneth, 2010). On assiste à des microtransformations dans le rapport à soi et à autrui. Ces altérations concernent aussi le rapport à l'école, aux enseignants, aux pairs et à la famille.

This article focuses on school dropouts and returners. Our purpose is to understand - thanks to the pupils' point of view - their perceived double experience of dropping out of and returning to school. With this in mind, data was collected using ethnographic methods to show the meaning subjects give to their "here and now". This data was interpreted with reference to symbolic interactionism and Axel Honneth's theory of recognition. We notice microtransformations in their relationships to themselves and others. These changes are reflected in their relationships with teachers, with fellow pupils and with their family.

\section{INDEX}

Mots-clés : décrochage provisoire, raccrochage, perception, reconnaissance

Keywords : temporary dropping out, returning to school, perception, recognition

\section{AUTEURS}

\section{LILIANE PELLETIER}

Maître de conférences, Sciences de l'éducation, Équipe Icare EA 7389

DRISS ALAOUI

Maître de conférences, HDR, Sciences de l'éducation, Équipe Icare EA 7389 